\title{
The Onset of Cosmic Ray Acceleration at Supernovae: From Shock Breakout to the First Decades
}

\author{
Gwenael Giacinti* \\ Max-Planck-Institut für Kernphysik, Heidelberg, Germany \\ University of Oxford, Clarendon Laboratory, Oxford, United Kingdom \\ E-mail: Giacinti@mpi-hd.mpg.de
}

\section{Anthony R. Bell}

University of Oxford, Clarendon Laboratory, Oxford, United Kingdom

\begin{abstract}
We demonstrate, for the first time, that CR acceleration can start significantly before shock breakout for some supernovae surrounded with optically thick winds. Diffusive shock acceleration notably requires the presence of a collisionless shock (CS). It is usually thought that the shock is initially radiation-dominated, and that the CS only forms in the optically thin layers of the wind. However, we show analytically and numerically, that a CS forms deep inside the thick layers of the wind for some astrophysically-relevant progenitors, such as possibly SN 2008D.

An observational consequence is that secondary $\mathrm{TeV}$ neutrinos can reach the observer up to $\sim 10$ hours before the first photons from shock breakout, enabling one to study the otherwise inaccessible optically thick layers of such winds [四].

We also investigate the beginning of cosmic ray (CR) acceleration at supernovae, from the first day to the first few decades following the explosion. We show that supernovae occuring in dense winds should accelerate $\mathrm{CR}$ protons to energies $E>\mathrm{PeV}[$ [ $]$. We present a study of the maximum CR energy.
\end{abstract}

The 34th International Cosmic Ray Conference,

30 July- 6 August, 2015

The Hague, The Netherlands

\footnotetext{
* Speaker.
} 


\section{Introduction}

Following core collapse in a massive star, a shock wave is launched. Inside the optically thick layers of the star, the shock is radiation-dominated, i.e. the radiation pressure in the downstream exceeds the fluid pressure [B]. Once the radiation-dominated shock (RDS) reaches the optically thin outer layers, photon escape ahead of the shock. This flash of photons corresponds to shock

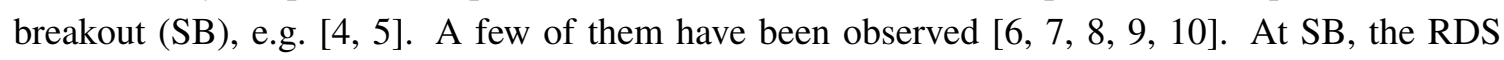
disappears and a collisionless shock (CS) later forms, e.g. [[], [1]. Diffusive shock acceleration may then become possible. A thorough understanding of the CS formation time is then crucial to study the onset of cosmic ray (CR) acceleration, when very high energies may be reached [D].

Some Wolf-Rayet (WR) stars, blue and red supergiants (RSG) may explode in optically thick winds. Ref. [[2] demonstrated that a CS must appear during or on the time scale of SB, when the RDS reaches the optically thin layers of the wind, at an optical depth $\tau \sim c / u_{\mathrm{s}}=\beta_{\mathrm{s}}^{-1}$ from the surface [[3]]. We demonstrate here that the formation of a CS occurs significantly before SB for some progenitors in thick winds. This redefines the onset of $\mathrm{CR}$ acceleration with respect to SB, since it can start in such cases significantly before SB. Supernova SN 2008D may have been an event in which a $\mathrm{CS}$ is formed before $\mathrm{SB}$, assuming a progenitor with the parameters derived in [1[4].

Finally, we show that supernovae occuring in dense winds are likely to accelerate CRs to $~$ $\mathrm{PeV}$ energies during the first few years or decades following the explosion.

\section{Collisionless shocks before breakout from optically thick winds}

For progenitors surrounded with optically thin winds, the flash of photons at SB accelerates the low density circumstellar medium to a velocity about $\propto r^{-2}$ ( $r$ is the distance to the center of the progenitor). The shocked outer layers of the star then push supersonically into the more slowly moving circumstellar material at larger $r$, which leads to the formation of a collisionless shock. We verified, with the 1D spherical radiation-hydrodynamics code presented in [四, that a CS forms after SB for a progenitor in an optically thin wind. In this case, the RDS stalls when entering optically thin material.

However, in some situations, the RDS can also stall inside optically thick material, and lead to the formation of a CS. We find that this happens for some supernovae exploding in optically thick winds. For thick winds, shock breakout occurs at a radius $r_{\mathrm{br}}$, which is larger than the radius of the star $r_{*}$. When the progenitor is surrounded with a very dense wind, such as for Type IIn SNe, the RDS survives the transition from the core to the thick wind at $r=r_{*}$. On the contrary, we find that when the wind is only moderately thick, the RDS stalls when exiting the hydrostatic core at $r=r_{*}$, and a CS forms in the wind at $r_{*}<r<r_{\mathrm{br}}$, before SB.

Let us consider two concentric shells in the thick wind, with respective radii $r_{1}(t)$ and $r_{2}(t)$, and velocities $u_{1}=u_{\mathrm{S}}$ and $u_{2}$, see Fig. . T. They are chosen such that $r_{1}(t=0)=r_{*}$, and $r_{*}<r_{2}(t=0)<$ $r_{*}+\lambda_{2} / \beta_{\mathrm{s}}$, where $\lambda_{2}$ is the photon mean free path in the wind at $r \simeq r_{*}$. In the limiting case where all photons that have accelerated the shell at $r_{1}$ also pass through the shell at $r_{2}$ (no absorption), the velocity reached by the latter shell cannot exceed $u_{2} \leq u_{1}\left(r_{*} / r_{2}(0)\right)^{2}+\kappa E_{\mathrm{em}} / 4 \pi c r_{2}^{2}(0)$, where $\kappa$ is the opacity and $E_{\mathrm{em}} \simeq \int_{r_{*}}^{r_{2}(0)} 4 \pi r^{2} \frac{1}{2 \kappa \lambda_{2}} u_{\mathrm{s}}^{2} d r$ is an upper limit on the energy that can be radiated 


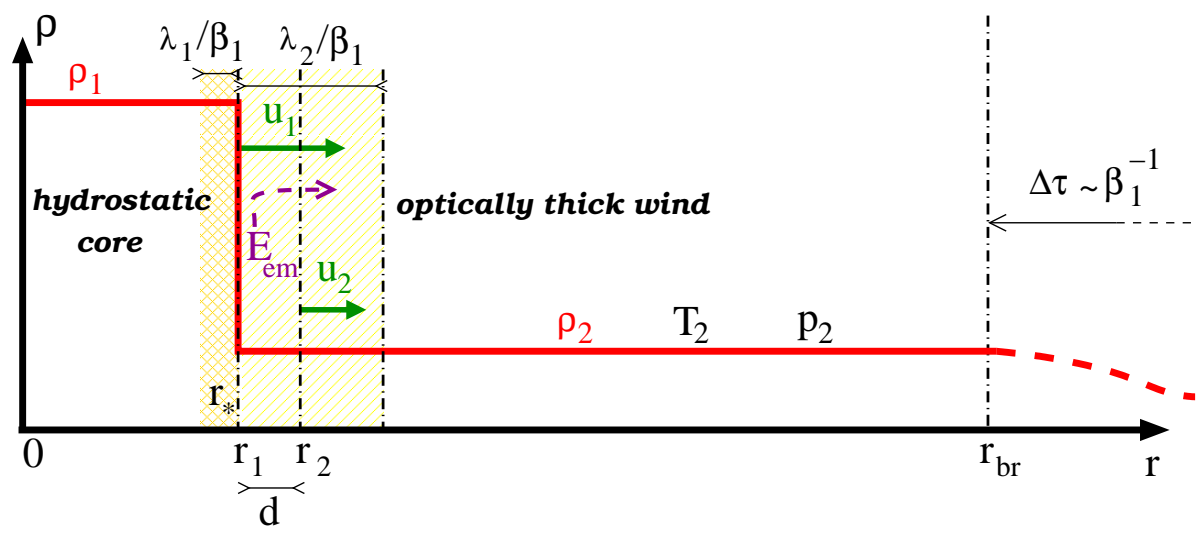

Figure 1: Toy model: The stellar core of radius $r_{*}=r_{1}(t=0)$ and density $\rho_{1}$ is surrounded with an optically thick wind with $\rho_{2}=1 / \kappa \lambda_{2}<\rho_{1}$. The RDS, with velocity $\sim u_{1}$ leaves the core at $t=0$. Shock breakout occurs at $r_{\mathrm{br}} \gg r_{*}$. A CS forms when the shell at $r_{1}(t)$ catches up supersonically that at $r_{2}(t)$, which can happen at $r \ll r_{\text {br }}$, see text.

through the shell at $r_{2}$ by the fluid between $r_{1}$ and $r_{2}$. If $E_{\mathrm{em}}$ is sufficiently large, it can compensate for the $\left(r_{*} / r_{2}(0)\right)^{2}$ factor : $u_{2}$ then remains $\geq u_{1}$ and the RDS survives in the wind. If the wind between the two shells cannot radiate enough photons through the shell at $r_{2}$ to compensate for the dilution of photons due to shock curvature, then the shell at $r_{1}$ can catch up and hit supersonically the shell at $r_{2}$, at a radius $r<r_{\mathrm{br}}$. A CS then forms before SB. One can show that this happens when [四]: $\beta_{\mathrm{s}} \lesssim 10 \lambda_{2} / r_{*}$. For a $r^{-2}$ wind, this condition becomes :

$$
\beta_{\mathrm{s}} \lesssim 0.1\left(\frac{u_{\mathrm{w}}}{10 \mathrm{~km} / \mathrm{s}}\right)\left(\frac{r_{*}}{10^{13} \mathrm{~cm}}\right)\left(\frac{5 \cdot 10^{-4} \mathrm{M}_{\odot} / \mathrm{yr}}{\dot{M}}\right),
$$

where $u_{\mathrm{w}}$ and $\dot{M}$ are respectively the wind velocity and the mass-loss rate of the progenitor. WolfRayet stars and red supergiants with relatively large $\dot{M}$ before the explosion are good candidates, but not progenitors of Type IIn SNe. Very interestingly, SN 2008D marginally satisfies this inequality with the parameters deduced in [ㅍ]]. For progenitors with wind densities $\propto r^{-2}$, this corresponds $r_{\mathrm{br}} \approx 10 r_{*}$. However, this scenario can also be satisfied for progenitors with $r_{\mathrm{br}} / r_{*} \gg 10$, if, for example, the wind density profile is flatter than $r^{-2}$ at $r<r_{\mathrm{br}}$, due to a change in the mass-loss rate with time.

We verified the above statements with our radiation-hydrodynamics code. Fig. [ shows the formation of a CS before breakout for a red supergiant exploding in a thick wind, see caption for details. Once formed, the CS continues to propagate to $r \gg r_{\mathrm{br}}$.

\section{Particle acceleration and high-energy neutrinos before and after shock breakout}

Assuming conservatively a magnetic field strength at the CS similar to that at the stellar surface, $B_{\mathrm{S}} \sim 10 \mathrm{G}$ [प]5], and wind densities $\rho \sim 10^{-11(-9)} \mathrm{g} \mathrm{cm}^{-3}$, the CS is super-Alfvénic. Once it is formed, CR acceleration may start. Coulomb losses for suprathermal particles are sufficiently small here and do not prevent them from entering diffusive shock acceleration and being accelerated. However, for WRs with $\dot{M} \gtrsim 10^{-3} \mathrm{M}_{\odot} \mathrm{yr}^{-1}$, such losses start to inhibit CR acceleration 

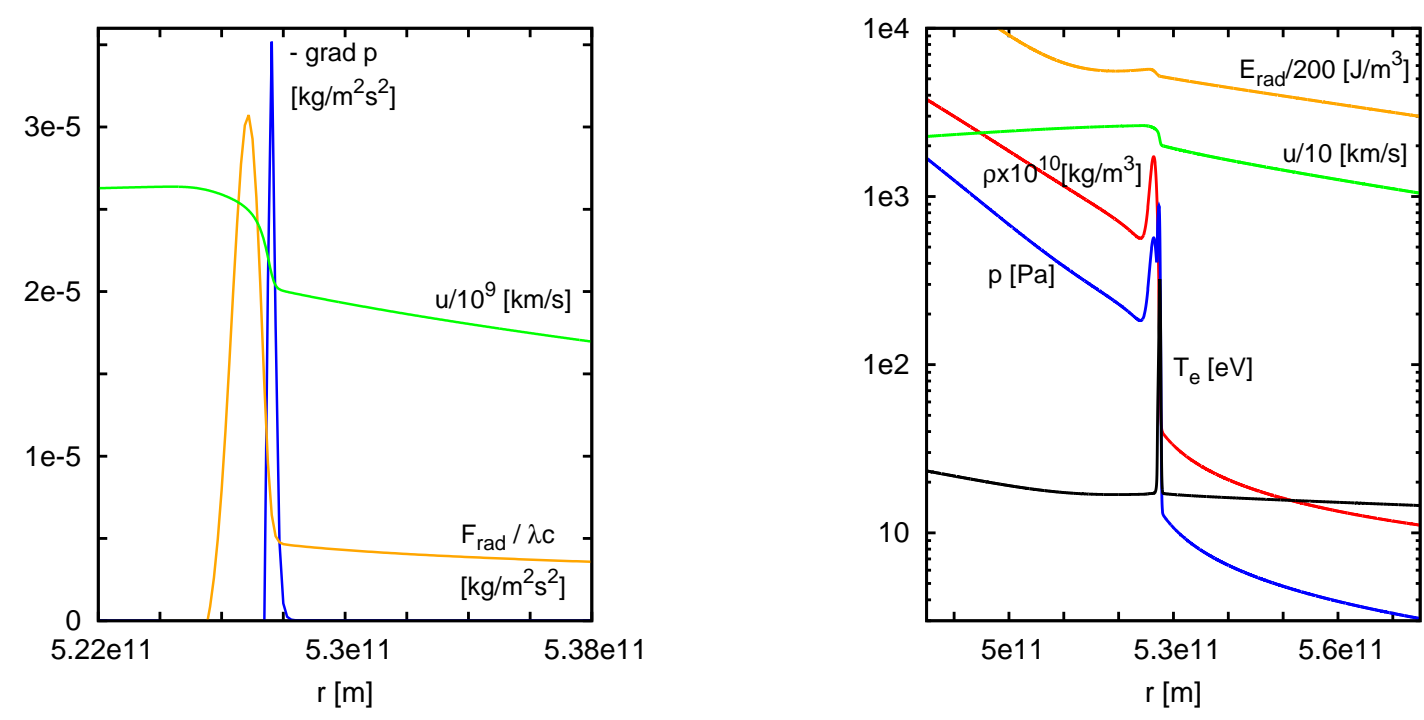

Figure 2: Simulation of a RSG explosion in a thick wind before breakout. Zoom around the downstream of the radiation-dominated transition of the shock, where a discontinuity (CS) can be seen around $r \approx 5.28 \times$ $10^{11} \mathrm{~m} \approx 1.6 r_{*} \approx r_{\mathrm{br}} / 2$. Left panel: Most of the fluid acceleration $d(\rho u) / d t$ is due to the radiation, except in a thin zone at the CS, where the fluid contribution dominates. Right panel: Peaks in $p$ and in the electron temperature $T_{\mathrm{e}}$ appear in the CS immediate downstream. $T_{\text {rad }}$ follows $T_{\mathrm{e}}$ except in the peak region.

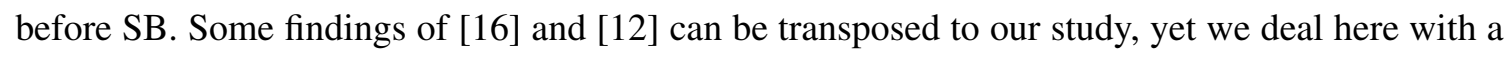
shock propagating in denser regions of the wind. Assuming Bohm diffusion for CRs at the CS [एT], and equal dwell times in the downstream and the upstream, one finds a typical acceleration time

$$
\tau_{\mathrm{CR}} \approx \frac{8 E_{\mathrm{CR}}}{3 e B_{\mathrm{s}} u_{\mathrm{s}}^{2}} \approx 30 \mathrm{~s}\left(\frac{E_{\mathrm{CR}}}{10 \mathrm{TeV}}\right)\left(\frac{B_{\mathrm{s}}}{10 \mathrm{G}}\right)^{-1}\left(\frac{\beta_{\mathrm{s}}}{0.1}\right)^{-2}
$$

for protons. This time can be optimistic when the discontinuity in velocity at the shock is still small due to smoothing by radiation. However, magnetic field amplification at the shock due to the non-resonant hybrid (NRH) instability [ए8] plays a role in the opposite direction by diminishing $\tau_{\mathrm{CR}}$ and thereby facilitating CR acceleration.

Let us note that $10 \mathrm{TeV}$ energies are reachable before breakout because $\tau_{\mathrm{CR}} \ll\left(r_{\mathrm{br}}-r_{*}\right) / u_{\mathrm{s}} \approx$ several hours (resp. minutes) for RSG (resp. WR) progenitors with $\beta_{\mathrm{s}}=0.1$ and $r_{\mathrm{br}} / r_{*} \approx 10$. For such RSGs, $\tau_{\mathrm{CR}}($ at $10 \mathrm{TeV})$ is smaller than energy loss times from pion production through inelastic $p p$ and $p \gamma$ collisions. The typical life time of a CR proton due to $p p$ collisions is

$$
\tau_{\mathrm{pp}} \approx 4 \min \left(\frac{u_{\mathrm{w}}}{10 \mathrm{~km} / \mathrm{s}}\right)\left(\frac{r}{10^{13} \mathrm{~cm}}\right)^{2}\left(\frac{\dot{M}}{5 \cdot 10^{-4} \mathrm{M}_{\odot} / \mathrm{yr}}\right)^{-1} .
$$

The background $\sim 10 \mathrm{eV}$ photons in the thick wind are not sufficiently energetic to trigger pion production through inelastic $p \gamma$ scattering. For $10 \mathrm{TeV}$ CRs, $\gtrsim 10 \mathrm{keV}$ photons are required to exceed the threshold for pion production. Photons with such energies can be produced by the radiative CS. However, the number density of target photons $n_{\gamma}$ must be much less than $\rho u_{\mathrm{S}}^{2} / h v$ [ㅁ] ]. 
We find for the typical life time of a CR proton due to $p \gamma$ collisions :

$$
\tau_{\mathrm{p} \gamma} \gtrsim 2 \min \left(\frac{u_{\mathrm{w}}}{10 \mathrm{~km} / \mathrm{s}}\right)\left(\frac{r}{10^{13} \mathrm{~cm}}\right)^{2}\left(\frac{\dot{M}}{5 \cdot 10^{-4} \mathrm{M}_{\odot} / \mathrm{yr}}\right)^{-1}\left(\frac{\beta_{\mathrm{s}}}{0.1}\right)^{-2}\left(\frac{E_{\mathrm{CR}}}{10 \mathrm{TeV}}\right)^{-1} .
$$

$e^{ \pm}$pair creation due to $p \gamma$ interactions does not yield a stronger constraint.

In the case of Wolf-Rayet progenitors with the above parameters, $\tau_{\mathrm{pp}, \mathrm{p} \gamma} \gtrsim 3 \mathrm{~s}$. Consequently, $\mathrm{TeV}$ energies may be reached for WRs.

Secondary $\gtrsim 100 \mathrm{GeV}-1 \mathrm{TeV}$ neutrinos (from notably $\pi^{ \pm}$decay) can then reach the observer before the first photons from breakout, when the CS forms before SB. The time interval between the arrival of the first neutrinos and photons is approximately

$$
\Delta t_{v \gamma} \approx \frac{r_{\mathrm{br}}-r_{*}}{c}\left(\frac{1}{\beta_{\mathrm{s}}}-1\right) \approx 8 \mathrm{hr}(\text { resp. } 5 \mathrm{~min})
$$

for RSGs (resp. WRs) with the above parameters, $r_{\mathrm{br}} / r_{*}=10$ and $\beta_{\mathrm{s}}=0.1$. Assuming that $5 \%$ of the energy processed by the shock is channelled into CRs, we typically find for a source at distance $l$, and a processed mass between $r_{*}$ and $r_{\mathrm{br}}$ of $\approx 10^{-5} \mathrm{M}_{\odot}$, that $\sim 10^{3}(3 \mathrm{kpc} / l)^{2}$ neutrinos with $\sim \mathrm{TeV}$ energies would be detectable before SB by IceCube or KM3NeT. One could record a few of such neutrinos for an event in the Magellanic Clouds.

\section{Particle acceleration at later times: First decades of a SN in a dense wind}

In this Section, we study CR acceleration at later times, after SB. Once the CS reaches a radius $r$ greater than a few $r_{\mathrm{br}}$, effects on the circumstellar wind from the flash of photons realeased at SB can be neglected.

It has been suggested since a long time that supernovae occuring in stellar wind cavities may accelerate CRs to very high energies [एT], and that radio supernovae may be sources of gammarays [ㅁ]]. Here, we study $\mathrm{CR}$ acceleration at $\mathrm{SNe}$ occuring in dense circumstellar winds, taking into account our latest understanding of magnetic field amplification at the shock. We find that, after a few days and during the first few weeks or decades following the explosion, cosmic rays accelerated at the shock may reach $\gtrsim \mathrm{PeV}$ energies.

Magnetic field amplification is (constantly) driven by the escape of the highest energy CRs in the upstream of the collisionless shock [ [ $]$. The growth rate of the fastest growing mode of the $\mathrm{NRH}$ instability is equal to $\gamma_{\max }=0.5 j_{\mathrm{CR}} \sqrt{\mu_{0} / \rho}$, where $j_{\mathrm{CR}} \simeq 0.03 \rho u_{\mathrm{s}}^{3} e / E_{\mathrm{CR}}$ is the CR current density which drives it, and the instability growth time is $\tau_{\mathrm{NRH}} \approx 5 \gamma_{\max }^{-1}$, see Reference [四] for more details. The condition for magnetic field amplification is then

$$
\mathscr{Q}_{\mathrm{CR}}=\int j_{\mathrm{CR}} \mathrm{d} t=10 \sqrt{\frac{\rho}{\mu_{0}}},
$$

where $\mathscr{Q}_{\mathrm{CR}}$ is the total electric charge of CRs passing through a unit surface area upstream of the shock, before the shock arrives.

Let us now assume a spherical supernova shock wave at radius $r(t)$, accelerating CRs to a maximum energy $E_{\mathrm{CR}}(r)$. The condition for CRs to be confined when the shock wave reaches 
radius $R$ reads :

$$
\int_{0}^{R} \frac{0.03 \rho(r) u_{\mathrm{s}}^{2}(r) e}{E_{\mathrm{CR}}(r)} r^{2} \mathrm{~d} r=10 R^{2} \sqrt{\frac{\rho(R)}{\mu_{0}}} .
$$

For a stellar mass-loss rate $\dot{M}$ and wind velocity $u_{\mathrm{w}}$, one has $\rho(R)=\dot{M} / 4 \pi u_{\mathrm{w}} R^{2}$. Differentiating Eqn. (4.2) with respect to $R$, one finds the following value for the maximum energy of CRs that can be acelerated at a supernova shock wave propagating in a circumstellar wind with density $\rho \propto r^{-2}:$

$$
E_{\mathrm{CR}} \approx 7 \mathrm{PeV}\left(\frac{u_{\mathrm{s}}}{30000 \mathrm{~km} \mathrm{~s}^{-1}}\right)^{2}\left(\frac{\dot{M}}{10^{-5} \mathrm{M}_{\odot} \mathrm{yr}^{-1}}\right)^{1 / 2}\left(\frac{u_{\mathrm{w}}}{10 \mathrm{~km} \mathrm{~s}^{-1}}\right)^{-1 / 2}
$$

This shows that $\mathrm{CRs}$ with $\mathrm{PeV}$ energies (if not even with energies beyond that of the knee, $\approx$ $4 \mathrm{PeV}$ ) should be accelerated during the first few weeks or decades following the explosion of stars occuring in dense circumstellar winds. A similar conclusion is reached by [2]].

It is interesting to note that the large magnetic field strengths inferred for radio supernovae (see e.g. Reference [2]]) may be a hint at strong magnetic field amplification due to the NRH instability from CR protons (and nuclei) being accelerated at the (forward) shock. Reference [23] studied radio supernova SN 1993J in detail and showed that the values of magnetic fields inferred from the radio data are roughly compatible with those expected from the NRH instability. It also found that SN 1993J should have been a PeVatron for about a decade.

\section{Conclusions}

During a core-collapse supernova, a radiation-dominated shock propagates through the progenitor star. If the surrounding wind is optically thin, this shock stalls when it reaches the outer layers of the stellar core and a collisionless shock forms in the wind.

For supernovae occuring in optically thick winds, the formation of a CS should also occur no later than during or on the time scale of shock breakout - from the 'outer' layers of the optically thick part of the wind.

We have demonstrated here that for some astrophysically-relevant progenitors surrounded with thick winds, a collisionless shock forms well before breakout, providing new ways to study invisible layers of their winds and to constrain stellar evolution theories. In such cases, the RDS has been found to stall when entering the optically thick part of the wind, notably because of shock curvature.

We have discussed, in Section [3, the onset of particle acceleration at the CS. For example, we predict that for some red supergiants surrounded with thick winds, a fraction of secondary high-energy neutrinos from CRs can arrive $\sim 10$ hours before photons from shock breakout.

We find that the CS forms after the RDS exits the core, and before breakout, for progenitors with shock velocities $\lesssim 0.1 \mathrm{c}\left(\frac{u_{\mathrm{w}}}{10 \mathrm{~km} / \mathrm{s}}\right)\left(\frac{\dot{M}}{5 \cdot 10^{-4} \mathrm{M}_{\odot} / \mathrm{yr}}\right)^{-1}\left(\frac{r_{*}}{10^{13} \mathrm{~cm}}\right)$, where $u_{\mathrm{w}}, \dot{M}$ and $r_{*}$ respectively denote the wind velocity, mass-loss rate and radius of the hydrostatic core. The wind has to be sufficiently dense to be optically thick but not excessively. Progenitors of Type IIn supernovae are expected to have too dense winds to form CS when the RDS leave their cores. However, Wolf-Rayet stars or red supergiants with either dense winds or enhanced mass-loss prior to the explosion are 
better candidates. For example, Type Ibc supernova SN 2008D/XRF 080109 has been interpreted by [14] as the explosion in a moderately thick wind of a WR star, undergoing an enhanced massloss during its last $\lesssim 10$ days. Interestingly, the parameters inferred by [4]] for SN 2008D make it marginally consistent with the above condition.

In Section 4, we have studied CR acceleration at significantly later times: From weeks to decades after SN shock breakout. We have shown that for SNe occuring in dense circumstellar winds, CRs can be accelerated up to a few $\mathrm{PeV}$, if not beyond the knee, for sufficiently fast shocks in sufficiently dense winds: $\sim 7 \mathrm{PeV}\left(\frac{u_{\mathrm{s}}}{30000 \mathrm{~km} \mathrm{~s}^{-1}}\right)^{2}\left(\frac{\dot{M}}{10^{-5} \mathrm{M}_{\odot} \mathrm{yr}^{-1}}\right)^{1 / 2}\left(\frac{u_{\mathrm{w}}}{10 \mathrm{kms}^{-1}}\right)^{-1 / 2}$. Studying CR acceleration in dense winds is important, because it should lead to a better understanding of the knee in the CR spectrum, see e.g. [24] and [D].

More generally, supernovae occurring in dense winds are promising targets for multi-messenger studies. The detection of their UVs, X-rays, $\gamma$-rays and TeV neutrinos will allow one to test a wide variety of physical and astrophysical phenomena in extreme conditions, such as particle acceleration, magnetic field amplification and shock physics.

\section{Acknowledgments}

This work was funded by the European Research Council under the European Community's Seventh Framework Programme (FP7/2007 - 2013) / ERC Grant agreement No. 247039.

\section{References}

[1] G. Giacinti and A. R. Bell, Collisionless Shocks and TeV Neutrinos before Supernova Shock Breakout from an Optically Thick Wind, MNRAS 449 (2015) 3693 [arXiv:1503.04170].

[2] A. R. Bell, K. Schure, B. Reville and G. Giacinti, Cosmic ray acceleration and escape from supernova remnants, MNRAS 431 (2013) 415 [arXiv:1301.7264].

[3] Y. B. Zel'dovich and Y. P. Raizer, Physics of shock waves and high-temperature hydrodynamic phenomena, Academic Press, New York 1966.

[4] S. A. Colgate, ApJ 187 (1974) 321.

[5] R. A. Chevalier and R. I. Klein, Nonequilibrium processes in the evolution of type II supernovae, ApJ 234 (1979) 597.

[6] S. Gezari, L. Dessart, S. Basa, D. C. Martin, J. D. Neill, S. E. Woosley, D. J. Hillier, P. Astier et al., Probing Shock Breakout with Serendipitous GALEX Detections of Two SNLS Type II-P Supernovae, ApJ 683 (2008) L131 [arXiv:0804.1123].

[7] K. Schawinski, S. Justham, C. Wolf, P. Podsiadlowski, M. Sullivan, K. C. Steenbrugge, T. Bell, H. -J. Roeser et al., Supernova shock breakout from a red supergiant, Science 321 (2008) 223 [arXiv:0803.3596].

[8] M. Modjaz, W. Li, N. Butler, R. Chornock, D. Perley, S. Blondin, J. S. Bloom, A. V. Filippenko et al., From shock breakout to peak and beyond: Extensive panchromatic observations of the aspherical type Ib supernova 2008D associated with Swift X-ray transient 080109, ApJ 702 (2009) 226

[arXiv:0805.2201]. 
[9] A. M. Soderberg, E. Berger, K. Page, P. Schady, J. Parrent, D. Pooley, X. -Y. Wang, E. Ofek et al., An extremely luminous X-ray outburst marking the birth of a normal supernova, Nature 453 (2008) 469 [arXiv:0802.1712].

[10] E. O. Ofek, I. Rabinak, J. D. Neill, I. Arcavi, S. B. Cenko, E. Waxman, S. R. Kulkarni, A. G. Yam et al., Supernova PTF 09uj: A possible shock breakout from a dense circumstellar wind, ApJ 724 (2010) 1396 [arXiv:1009.5378].

[11] L. Ensman and A. Burrows, Shock breakout in SN 1987A, ApJ 393 (1992) 742.

[12] B. Katz, N. Sapir and E. Waxman, X-rays, gamma-rays and neutrinos from collisionless shocks in supernova wind breakouts, Proc. of the International Astronomical Union 7 (2011) 274 [arXiv:1106.1898].

[13] B. Katz, R. Budnik and E. Waxman, Fast radiation mediated shocks and supernova shock breakouts, ApJ 716 (2010) 781 [arXiv:0902.4708].

[14] G. Svirski and E. Nakar, SN 2008D: A Wolf-Rayet explosion through a thick wind, ApJ 788 (2014) L14 [arXiv:1403.3400].

[15] R. Barvainis, G. McIntoch and C. R. Predmore, Nature 329 (1987) 613.

[16] E. Waxman and A. Loeb, TeV neutrinos and GeV photons from shock breakout in supernovae, Phys. Rev. Lett. 87 (2001) 071101 [astro-ph/0102317].

[17] B. Reville and A. R. Bell, Universal behaviour of shock precursors in the presence of efficient cosmic-ray acceleration, MNRAS 430 (2013) 2873 [arXiv:1301.3173].

[18] A. R. Bell, Turbulent amplification of magnetic field and diffusive shock acceleration of cosmic rays, MNRAS 353 (2004) 550.

[19] H. J. Voelk and P. L. Biermann, Maximum energy of cosmic-ray particles accelerated by supernova remnant shocks in stellar wind cavities, ApJ 333 (1988) L65.

[20] J. G. Kirk, P. Duffy and L. Ball, Radio supernovae as TeV gamma-ray sources, Astron. Astrophys. 293 (1995) L37.

[21] M. Cardillo, E. Amato and P. Blasi, On the cosmic ray spectrum from type II Supernovae expanding in their red giant presupernova wind, Astropart. Phys. 69 (2015) 1 [arXiv:1503.03001].

[22] C. Fransson and C.-I. Björnsson, Radio Emission and Particle Acceleration in SN 1993J, ApJ 509 (1998) 861 [astro-ph/9807030].

[23] V. Tatischeff, Radio emission and nonlinear diffusive shock acceleration of cosmic rays in the supernova SN 1993J, Astron. Astrophys. 499 (2009) 191 [arXiv:0903.2944].

[24] L. G. Sveshnikova, The knee in galactic cosmic ray spectrum and variety in supernovae, Astron. Astrophys. 409 (2003) 799 [astro-ph/0303159]. 